\title{
On the QCD phase structure from effective models
}

\author{
Bernd-Jochen Schaefer ${ }^{1}$ and Mathias Wagner ${ }^{2}$ \\ ${ }^{1}$ Institut für Physik, Karl-Franzens-Universität Graz, Austria \\ ${ }^{2}$ Institut für Kernphysik, Technische Universität Darmstadt, Germany
}

October 22, 2018

\begin{abstract}
Some recent theoretical developments of the QCD phase diagram are summarized. Chiral symmetry restoration and the confinement/deconfinement transition at nonzero temperature and quark densities are analyzed in the framework of an effective linear sigma model with three light quark flavors. The sensitivity of the chiral transition as well as the existence of a critical end point in the phase diagram on the value of the sigma mass is explored. The influence of the axial anomaly on the chiral critical surface is addressed. Finally, the modifications by the inclusion of the Polyakov loop on the phase structure are investigated.
\end{abstract}

\section{Introduction}

In recent years the phase diagram of strongly interacting matter has become the focus of theoretical and experimental attention. It is believed that the underlying fundamental theory of the strong interaction is described by Quantum Chromodynamics (QCD). At finite temperature and finite densities QCD predicts two different phase transitions which are associated with two opposite quark mass limits (for reviews see e.g. [1, 2]).

For vanishing quark masses, i.e., in the chiral limit, QCD has an exact global $U\left(N_{f}\right)_{L} \times U\left(N_{f}\right)_{R}$ chiral symmetry, where $N_{f}$ denotes the number of quark flavors. The axial $U(1)_{A}$ anomaly, induced by instantons, breaks the axial $U\left(N_{f}\right)_{A}$ part of the chiral symmetry explicitly to $S U\left(N_{f}\right)_{A}$. In addition, in the vacuum the $S U\left(N_{f}\right)_{A}$ is spontaneously broken by a finite expectation value of the quark condensate $\langle\bar{q} q\rangle \neq 0$. As a consequence of the Goldstone theorem, $N_{f}^{2}-1$ massless pseudoscalar Goldstone bosons are expected to emerge. For $N_{f}=2$ the associated Goldstone bosons are the three pseudoscalar pions and for $N_{f}=3$ one has additionally the four kaons and the pseudoscalar $\eta$ meson which together with the pions constitute the pseudoscalar meson octet. Once the quark masses obtain finite values, i.e., leaving the chiral limit, chiral symmetry is broken explicitly and all these Goldstone bosons acquire masses as measurable in the experiment. However, at high temperatures and densities this symmetry breaking pattern changes drastically. In hot and dense matter the $S U\left(N_{f}\right)_{A}$ symmetry and additionally, if instantons are sufficiently screened, the explicitly broken axial $U(1)_{A}$ symmetry will become restored again. As a consequence, the masses of the pseudoscalar Goldstone bosons will degenerate with the masses of the corresponding chiral scalar partners, signaling in this way the restoration of chiral symmetry. The associated phase transition is commonly referred to as the chiral phase transition. 

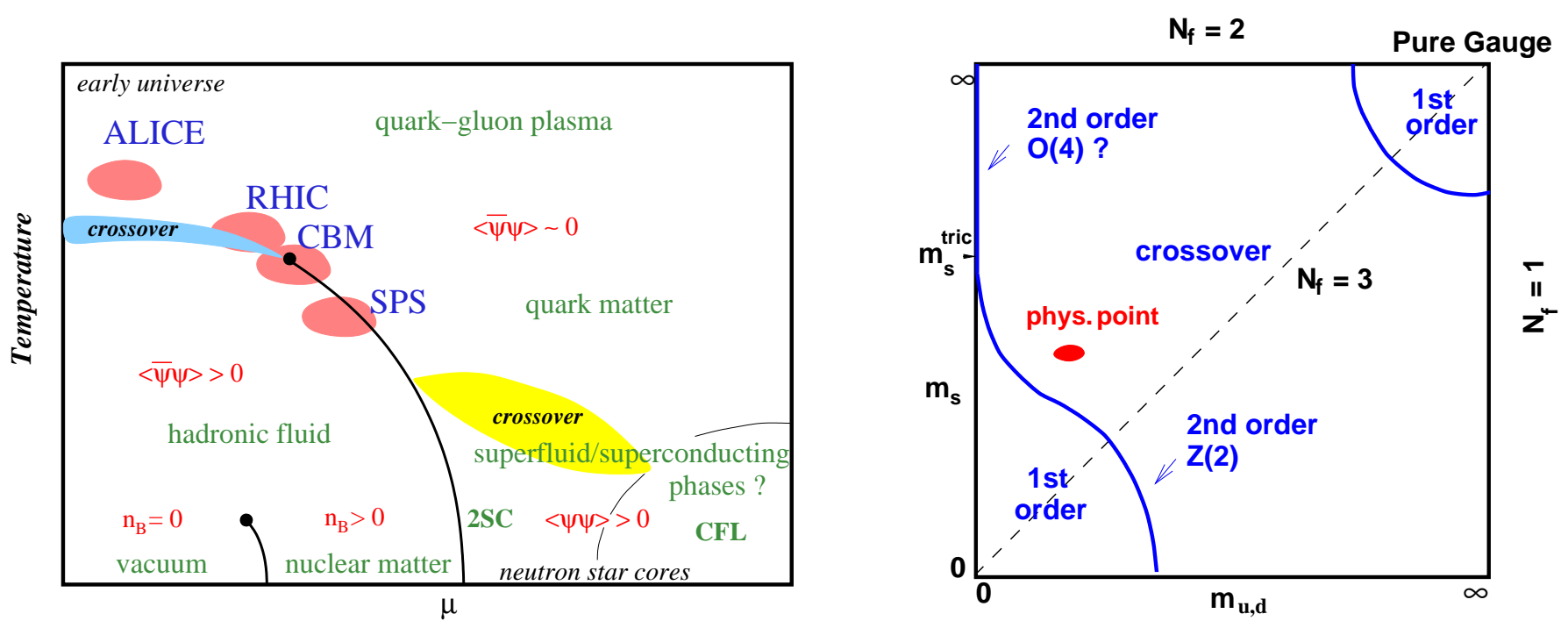

Figure 1: Schematic phase transition behavior of $N_{f}=2+1$ flavor QCD in the $(T, \mu)$ plane (left panel) and for vanishing chemical potential in the $\left(m_{u, d}, m_{s}\right)$ quark mass plane (right panel) [4].

In the opposite quark mass limit, the so-called quenched limit of QCD with infinitely heavy quark masses, QCD reduces to a pure $S U\left(N_{c}\right)$ gauge theory which is invariant under a global $Z\left(N_{c}\right)$ center symmetry. In contrast to the chiral symmetry, the center symmetry is spontaneously broken at high temperatures and densities, i.e., in the color deconfined quark-gluon plasma phase and is restored in the hadronic phase at small temperatures and densities. The associated phase transition from the hadronic (glueball) phase to the color deconfined plasma phase is the confinement/deconfinement phase transition. The center symmetry is always broken explicitly when dynamical quarks are present, i.e., when the quenched limit of QCD is left.

Both phase transitions are conceptually distinct phenomena of QCD. For the experiment it is important to investigate and understand the interplay between these phase transitions, in particular, for realistic quark masses. Based on theoretical models and QCD lattice simulations a generic phase diagram for $N_{f}=2+1$ quark flavors in the temperature and (baryo)chemical potential $\mu$ plane can be drawn as in the left panel of Fig. 1. Here not only the chiral and deconfinement phase transition from the hadronic fluid phase to the quark-gluon plasma are shown. The regions probed by some already running or planned relativistic heavy-ion collision experiments such as ALICE, RHIC, CBM and SPS are also marked in the figure. So far, it is still an open issue whether both phase transitions, the chiral and deconfinement transition, take place at the same temperatures and densities yielding thus a single transition or crossover line in the QCD phase diagram as indicated in the figure. For example, McLerran and Pisarksi suggested that this is not the case at moderate temperature and large chemical potential. Based on large- $N_{c}$ arguments, they concluded that in this phase diagram region there might be a new, so-called quarkyonic phase which is still confining but chirally symmetric [3]

The situation is even more sophisticated since some properties of the chiral phase transition such as its order depend on $N_{f}$ and the strength of the axial anomaly. The status for vanishing chemical potential is partly summarized in the right panel of Fig. 1, in the limiting case of two massless light quarks, $m_{u, d}=0$, and an infinite strange quark mass $m_{s}$, which corresponds to $N_{f}=2$, it is conjectured that the finite temperature chiral phase transition is of second-order for a constant anomaly strength and lies in the universality class of the Heisenberg $O(4)$ model in three dimensions.

If the anomaly strength is identified with the instanton density, a temperature-dependent strength of the axial anomaly would arise. It is supposed that the strength vanishes at high temperatures. This 
temperature-dependent axial anomaly can change the chiral transition to first-order.

The chiral transition will also be of first-order once the strange quark mass drops below a certain critical value. This critical mass value is a tricritical end point of the second-order transition line. For three vanishing quark masses the first-order transition has been confirmed by a renormalization group analysis and is independent of the strength of the axial anomaly [5]. The first-order region in the $\left(m_{u, d}, m_{s}\right)$ plane still persists for small light and strange quark masses and is finally terminated by a second-order transition boundary. This boundary line separates the first-order region from the crossover region in the $\left(m_{u, d}, m_{s}\right)$ plane. QCD lattice simulations indicate that the physical mass point, labeled as a red point in the Fig. 1, is located in the crossover regime. In contrast to the boundary line at $m_{u, d}=0$, which lies presumably in the $O(4)$ universality class, the universality class changes for finite $m_{u, d}$ to the one of the $Z(2)$ three-dimensional Ising model.

For finite chemical potential the area of the first-order regime in the quark mass plane also changes. If it grows for increasing chemical potential the boundary may hit and pass over the position of the physical mass point, turning the chiral phase transition from a crossover to a second-order or first-order transition and as a consequence, the existence of critical end point (CEP) in the QCD phase diagram becomes possible. This scenario is denoted as the standard one.

On the other hand, if the first-order region shrinks for increasing chemical potential the chiral phase transition sticks to be a crossover and the existence of a CEP in the phase diagram is not possible, which is commonly labeled as the non-standard scenario and has been predicted by de Forcrand and Philipsen [6].

The existence or exclusion of a QCD critical end point has not yet been confirmed by QCD lattice simulations. At finite chemical potential these Monte-Carlo simulations suffer from the notorious sign problem because the quark determinant in the QCD partition function becomes a complex quantity which entangles its probability interpretation. But recently, some progress could be achieved in extrapolating zero chemical potential Monte-Carlo simulations to finite chemical potentials [7]. However, all these extrapolation techniques are still limited to small chemical potentials. Furthermore, both above mentioned scenarios, the standard and the non-standard ones, are seen on the lattice with different extrapolation techniques.

So far, only model studies give direct and indirect evidences for the existence of the critical end point in the whole phase diagram. But they cannot predict its precise location. The location and even its existence depend also on the magnitude of the axial anomaly and on vector-channel interactions [8, 9]. This is plausible because the zeroth-component of the vector-channel interaction is directly coupled to the quark density which surely modifies the interactions in the finite-density environment. Due to the repulsive vector-vector interaction the first-order transition is weakened. As a consequence a standard scenario could change to a non-standard one depending, of course, on the strength of the vector coupling.

In all, the influence of the axial anomaly and also of the vector-vector interaction on the QCD phase structure is of relevance and should be investigated in a quantitative manner. Moreover, in order to bridge the gap between existing lattice data at zero chemical potential and interesting regions in the QCD phase diagram at finite chemical potential effective models are useful and inevitable tools. They share the relevant symmetries with the underlying QCD and allow the investigations in a simplified framework. One example of such effective models is the chiral quark-meson model which allows to explore the chiral phase transition, see e.g. [10].

\section{Chiral effective quark-meson models}

Based on a previous analysis within an effective quark-meson model with two quark flavors [11, 12, 13] an extension to three quark flavors is done straightforwardly which enables the investigation of the 

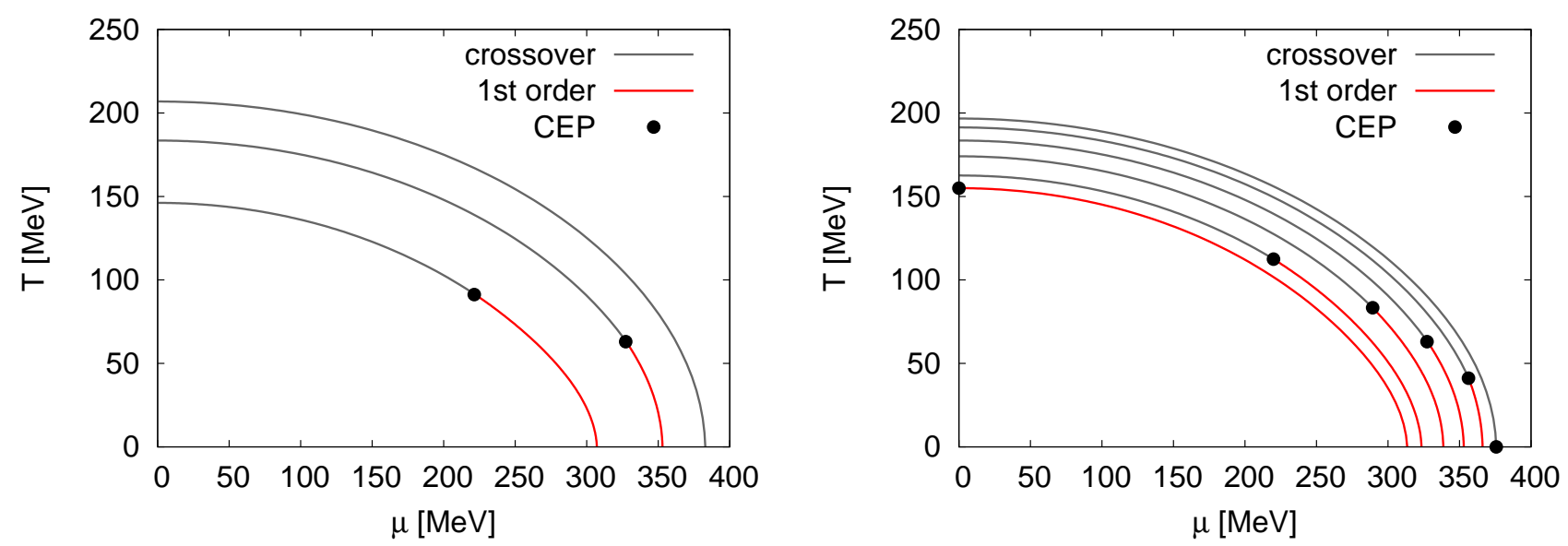

Figure 2: Left: Phase diagrams with axial anomaly for different values of the sigma mass: $m_{\sigma}=600$ $\mathrm{MeV}$ (lower lines), $800 \mathrm{MeV}$ and $900 \mathrm{MeV}$ (upper line). Right: Phase diagrams with axial anomaly for $m_{\sigma}=800 \mathrm{MeV}$ but different pion masses $m_{\pi} / m_{\pi}^{*}=0.49$ (lower line), 0.6, 0.8, 1.0, 1.2, 1.36 (upper line) where the ratio $m_{\pi} / m_{K}=m_{\pi}^{*} / m_{K}^{*}$ is kept fixed with $m_{\pi}^{*}=138 \mathrm{MeV}$ and $m_{K}^{*}=496 \mathrm{MeV}$.

chiral $S U(3) \times S U(3)$ symmetry restoration with temperature and quark chemical potential including the axial $U(1)_{A}$ anomaly. Here we briefly summarize some results of Ref. [14] where the three-flavor quark-meson model has been treated in mean-field approximation. In this approximation the quantum and thermal meson fluctuations of the grand potential are neglected while the quarks/antiquarks are retained as quantum fields. The resulting integration over the Grassmann fields yields finally the $T$ and $\mu$-dependent quark/antiquark contribution $\Omega_{\bar{q} q}(T, \mu)$ of the grand potential wherein the ultraviolet divergent vacuum contribution has been neglected. After all the total grand potential is a sum of $\Omega_{\bar{q} q}(T, \mu)$ and a meson potential $U\left(\sigma_{x}, \sigma_{y}\right)$,

$$
\Omega(T, \mu)=\Omega_{\bar{q} q}(T, \mu)+U\left(\sigma_{x}, \sigma_{y}\right),
$$

where $\sigma_{x}$ and $\sigma_{y}$ denote the nonstrange and strange condensates, respectively. The condensates are the corresponding chiral order parameters and depend on $T$ and $\mu$. Note, that the quark contribution also depends on these condensates implicitly via the quark masses. Since we consider symmetric quark matter a uniform quark chemical potential has been introduced.

The resulting phase diagrams with explicit $U(1)_{A}$ symmetry breaking for three different values of $m_{\sigma}$ are shown in the left panel of Fig. 2, For certain values of $m_{\sigma}$ a CEP is found. Compared to recent lattice simulations, this point is located at smaller temperatures and larger chemical potentials. Anyhow, its exact location in the phase diagram cannot be predicted by effective models. The mass of the $\sigma$ meson is one model input parameter which is poorly known experimentally. We therefore use different input values for $m_{\sigma}$ in order to study its mass dependence. One sees for increasing $m_{\sigma}$ that the location of the CEP moves towards the $\mu$-axis. Already for $m_{\sigma}=900 \mathrm{MeV}$ the CEP disappears and the chiral phase transition is a smooth crossover over the entire phase diagram. Without axial anomaly almost no difference of the phase boundary and hence of the location of the CEP is seen.

In Ref. [8] a gauged chiral $U(2) \times U(2)$ symmetric linear sigma model without quarks within the 2PI resummation scheme has been considered. If the influence of the vector mesons are neglected the opposite behavior of the chiral transition as a function of $m_{\sigma}$ is observed: at $\mu=0$ a crossover is found for a small $\sigma$ mass and a first-order transition for a large $\sigma$ mass. On the other hand, if vector mesons are incorporated the transition leads to a more rapid crossover and brings one closer to the second-order critical point. 

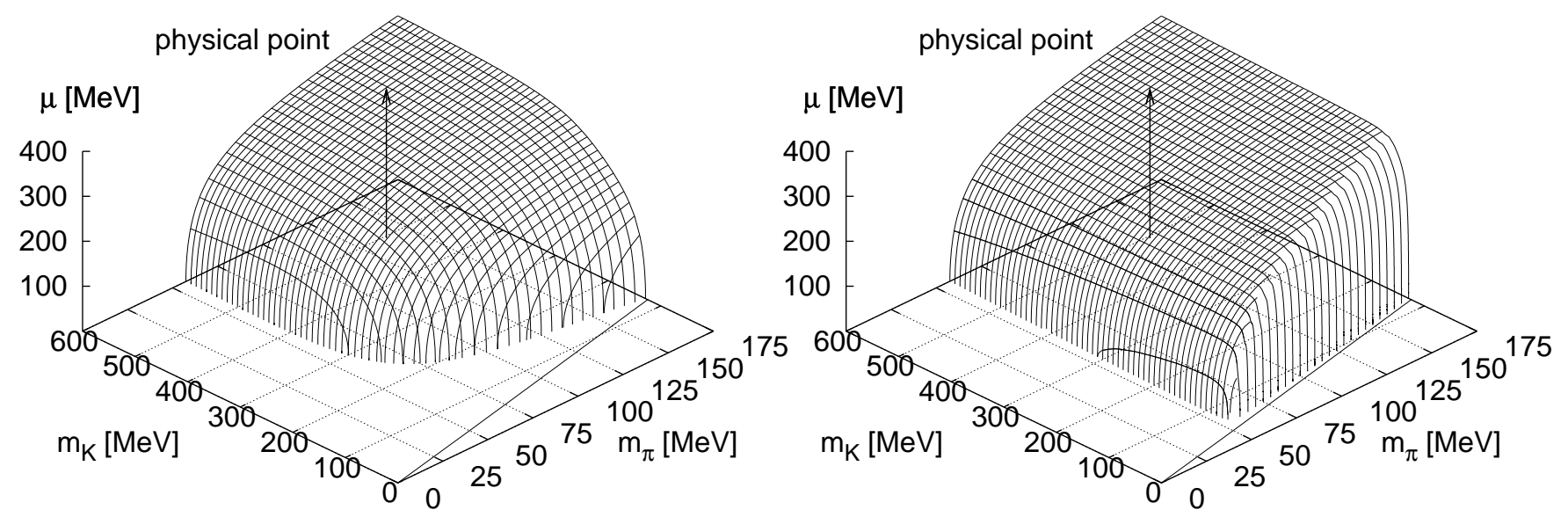

Figure 3: Chiral critical surface as a function of the pion and kaon masses for $m_{\sigma}=800 \mathrm{MeV}$ with (left) and without axial anomaly (right). The arrow points to the critical quark chemical potential at realistic pion and meson masses and is denoted as physical point.

Independent of the $U(1)_{A}$ symmetry breaking a first-order phase transition in the chiral limit is expected. This behavior is shown in the right panel of Fig. 2 where the phase diagrams including the anomaly for varying pion and kaon masses are shown for $m_{\sigma}=800 \mathrm{MeV}$. For this figure a path in the $\left(m_{\pi}, m_{K}\right)$-plane through the physical mass point towards the chiral limit has been chosen by varying the pion mass while keeping the ratio $m_{\pi} / m_{K}$ fixed. On the one hand, for a pion mass 1.36 times larger than the physical one, the CEP lies exactly on the $\mu$-axis (for $m_{\sigma}=800 \mathrm{MeV}$ ) and the chiral transition is a smooth crossover over the entire phase diagram. On the other hand, for decreasing pion masses the location of the CEP moves towards the $T$-axis and for a pion mass below half of the physical one the chiral transition turns into a first-order one for all densities and no CEP exists any longer.

This behavior of the CEP excludes the nonstandard scenario described in the introduction. The chiral critical surface which is defined by the value of the critical chemical potential of the CEP for a given mass pair $\left(m_{\pi}, m_{K}\right)$, is evaluated in Fig. 3 as a function of the pion and the kaon masses with (left) and without (right) $U(1)_{A}$ symmetry breaking. For values of the chemical potential above the surface the chiral transition is of first-order while for values below the surface the transition lies in the crossover region. With or without anomaly the surface grows out perpendicular from the mass plane at $\mu=0$ and the tangent plane to the critical surface has a decreasing slope for larger masses. Consequently, the first-order region grows for increasing chemical potentials. Since the critical chemical potential cannot grow arbitrarily the surface must have a boundary at larger pion and kaon masses which is not shown in the figure.

Furthermore, the effect of the $U(1)_{A}$ anomaly on the shape of the surface is rather marginal for kaon masses greater than $400 \mathrm{MeV}$. This is reasonable since for larger kaon masses the strange sector decouples effectively from the light nonstrange sector and the chiral transition is basically driven by the light nonstrange particles. For kaon masses smaller than $400 \mathrm{MeV}$ a considerable influence of the anomaly on the shape of the critical surface is seen: without anomaly the region of first-order phase transitions at $\mu=0$ is considerably reduced. 

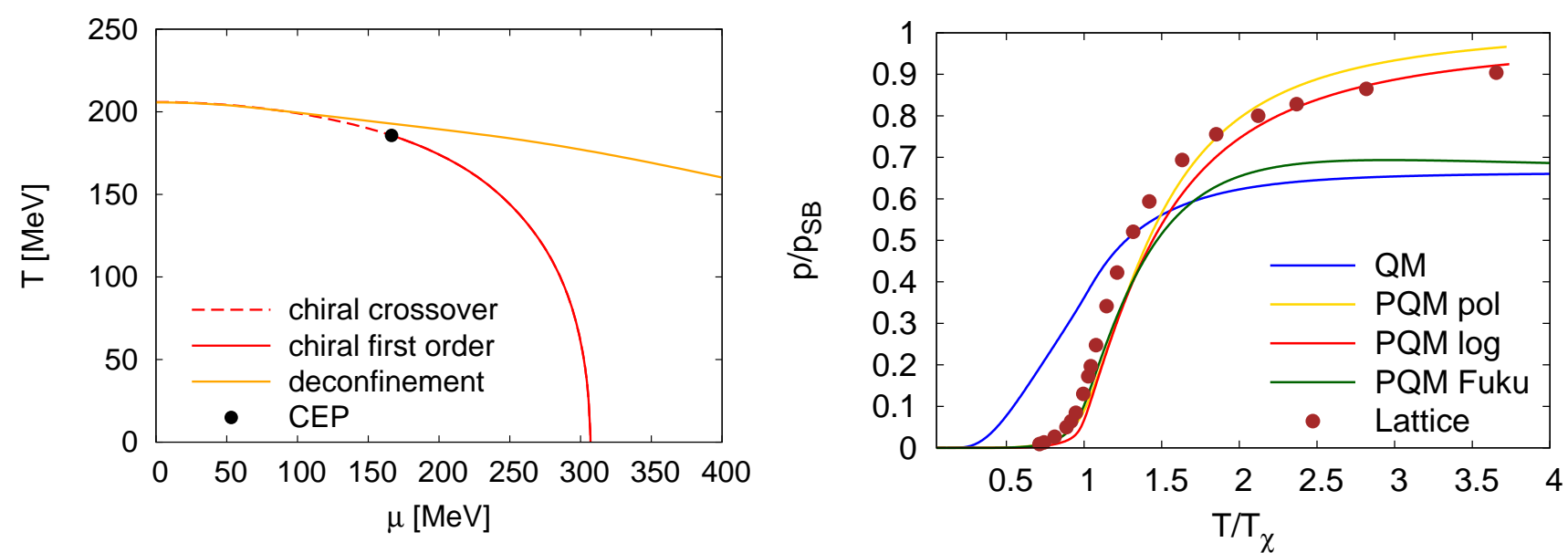

Figure 4: Left: Phase diagram for the three flavor PQM model with a logarithmic Polyakov loop potential. Right: Scaled pressure for the PQM model for three different Polyakov loop potentials (labeled as pol, log and Fuku) in comparison with recent lattice data for $2+1$ flavors [15]. The scaled pressure without the Polyakov loop dynamics (QM) is also shown.

\section{Emulating the Polyakov-loop dynamics}

So far only the chiral phase transition has been considered. Recently, the quark-meson model could be combined with the Polyakov loop which allows to investigate both, the chiral and the deconfinement phase transition. In general, the Polyakov loop $\Phi$ is a complex scalar field and serves as an order parameter for the confinement/deconfinement transition in the quenched limit. Since it is related to the free energy of a static test quark, it vanishes in the confined phase where the free energy of a single quark diverges and takes a finite value in the deconfined phase. It is linked to the $Z\left(N_{c}\right)$ center symmetry of the $S U\left(N_{c}\right)$ gauge group. Thus, the confining phase is center symmetric, whereas the center symmetry is spontaneously broken in the deconfined phase.

In the presence of dynamical quarks and nonvanishing chemical potential it is not clear whether the Polyakov loop still serves as an order parameter. In this case, the free energy does not diverge anymore and the order parameter is always nonzero. Because the free energies of quarks and antiquarks are different in the medium, $\Phi$, related to quarks, and the Hermitian (charge) conjugate $\bar{\Phi}$, related to antiquarks, will differ.

In pure Yang-Mills theory the real mean values $\Phi$ and $\bar{\Phi}$ are given by the minima of an effective Polyakov loop potential $\mathcal{U}(\Phi, \bar{\Phi})$ which can be constructed from lattice data. Finally, the dynamical quark sector of QCD is included by coupling the Polyakov loop to the quark sector of the quark-meson model which leads to the Polyakov-quark-meson (PQM) model with an interaction potential between quarks, mesons and the Polyakov loop variables. For two quark flavors the PQM model has been introduced in [16], wherein a polynomial ansatz for the Polyakov loop potential has been used. Here, we extend the two flavor PQM model to three quark flavors together with three different realizations of the Polyakov loop potential $\mathcal{U}(\Phi, \bar{\Phi})[17,16,18$.

For three quark flavors the total grand potential of the PQM model is a sum of three contributions

$$
\Omega(T, \mu)=\Omega_{\bar{q} q}(T, \mu ; \Phi, \bar{\Phi})+U\left(\sigma_{x}, \sigma_{y}\right)+\mathcal{U}(T ; \Phi, \bar{\Phi})
$$

where the quark/antiquark contribution $\Omega_{\bar{q} q}$ is modified by the Polyakov loop variables. The mesonic contribution $U\left(\sigma_{x}, \sigma_{y}\right)$ is the same as for the quark-meson model, Eq. (11). 
In Fig. 4 (left panel) the resulting phase diagram of the PQM model with three quark flavors for a logarithmic Polyakov loop potential, adopted from [18], is shown. The phase boundaries are extracted from the peak in the temperature derivative of the corresponding light nonstrange condensate and the Polyakov loop variable. The model parameters are adjusted in such a way that both phase transitions, the chiral and the deconfinement transition, coincide at $\mu=0$ as is indicated by recent lattice simulations [19]. In general, the chiral transition temperature is shifted to higher values if the Polyakov loop is included and a more rapid crossover is observed.

For increasing chemical potential both transition still coincide initially but then start to deviate before the chiral critical point is reached. The chiral transition always occurs below the deconfinement transition. For small temperatures the chiral transition becomes of first-order and both transitions are well separated. This separation might be related to the existence of a quarkyonic phase but work in this direction is still in progress.

The coupling of the quark dynamics to the Polyakov loop improves the equation of state in the chirally broken phase at low temperatures and densities. This is demonstrated in Fig. 4 (right panel) where the scaled pressure, normalized to the Stefan-Boltzmann pressure, is shown at $\mu=0$ for three different realizations of the Polyakov loop potential. In comparison, recent $2+1$-flavor lattice data for $N_{\tau}=6$ [15] are also included and are in reasonable agreement with our results. The suppression of the quark contribution in the confined phase is clearly visible compared to the pure QM model calculation.

\section{Acknowledgments}

BJS is grateful to the organizers of the 30th International School of Nuclear Physics in Erice, Italy, for

the invitation and acknowledges the European Physical Society (EPS) for the scholarship. MW was supported by the BMBF grant 06DA123.

\section{References}

[1] H. Meyer-Ortmanns, Rev. Mod. Phys. 68, 473 (1996).

[2] D. H. Rischke, Prog. Part. Nucl. Phys. 52, 197 (2004).

[3] L. McLerran and R. D. Pisarski, Nucl. Phys. A796, 83 (2007).

[4] F. R. Brown et al., Phys. Rev. Lett. 61, 2058 (1988).

[5] R. D. Pisarski and F. Wilczek, Phys. Rev. D29, 338 (1984).

[6] P. de Forcrand and O. Philipsen, Nucl. Phys. B642, 290 (2002).

[7] C. Schmidt, PoS LAT2006, 021 (2006).

[8] S. Strueber and D. H. Rischke, Phys. Rev. D77, 085004 (2008).

[9] K. Fukushima, arXiv:0809.3080 [hep-ph].

[10] B.-J. Schaefer and J. Wambach, Phys. Part. Nucl. 39, 1025 (2008).

[11] B.-J. Schaefer, PoS CPOD07, 032 (2007).

[12] B.-J. Schaefer and J. Wambach, Phys. Rev. D75, 085015 (2007).

[13] B.-J. Schaefer and J.Wambach, Nucl. Phys. A757, 479 (2005).

[14] B.-J. Schaefer and M. Wagner, arXiv:0808.1491 [hep-ph].

[15] M. Cheng et al., Phys. Rev. D77, 014511 (2008).

[16] B.-J. Schaefer, J. M. Pawlowski, and J. Wambach, Phys. Rev. D76, 074023 (2007).

[17] K. Fukushima,Phys. Rev. D77, 114028 (2008).

[18] S. Roessner, C. Ratti, and W. Weise, Phys. Rev. D75, 034007 (2007).

[19] M. Cheng et al., Phys. Rev. D74, 054507 (2006). 